\title{
Epidemiological Profile of Hispanics Admitted With Acute Myocardial Infarction in Puerto Rico: The Experience of 2007, 2009 and 2011
}

\author{
Dagmar F. Hernandez-Suarez ${ }^{\mathrm{a}}$, Anthony D. Osterman-Pla ${ }^{\mathrm{a}}$, Onelys Carrasquillo ${ }^{\mathrm{a}}$, \\ Juan Aranda ${ }^{\text {a }}$, Stella Baez ${ }^{\mathrm{a}}$, Mariel Lopez ${ }^{\mathrm{b}}$, Enid J. Garcia-Rivera ${ }^{\mathrm{b}, \mathrm{c}}$
}

\begin{abstract}
Background: A limited number of studies have been published about coronary artery disease in Hispanics, particularly among the Puerto Rican population. The aim of this study was to present a clinical epidemiological profile and management practices in patients hospitalized in Puerto Rico with acute myocardial infarction (AMI).

Methods: This secondary data analysis from the Puerto Rico Cardiovascular Surveillance Study included 6,162 patients at 19 hospitals in Puerto Rico, during years 2007, 2009 and 2011.

Results: The mean age of the patients diagnosed with AMI was $67 \pm$ 13.6 years old, with women being older than men $(\mathrm{P}<0.001)$. Women had a different risk factor burden when compared to men. Car/walked in was the principal mode of hospital transportation (65.9\%). Women received less medications and cardiac procedures when compared to men. While no significant differences in length of hospital stay (LOS) were observed between genders, in-hospital mortality rate was higher in females when compared with males $(6.5 \%$ vs. $4.5 \%$; $\mathrm{P}<0.001)$.
\end{abstract}

Conclusion: Prompt initiatives should be implemented to raise awareness, reduce gender disparities and improve outcomes in patients hospitalized with an AMI in Puerto Rico.

Keywords: Acute myocardial infarction; Hispanics; Incidence; Population surveillance

\section{Introduction}

Cardiovascular diseases (CVDs) are the leading cause of

Manuscript accepted for publication January 26, 2017

a Department of Medicine, School of Medicine, University of Puerto Rico Medical Science Campus, San Juan, Puerto Rico

bUniversity of Puerto Rico Endowed Health Services Research Center, San Juan, Puerto Rico

${ }^{\mathrm{c}}$ Corresponding Author: Enid J. Garcia-Rivera, Endowed Health Services Research Center, University of Puerto Rico School of Medicine, Medical Sciences Building, PO Box 365067, San Juan 00936-5067, Puerto Rico.

Email: enid.garcia3@upr.edu

doi: https://doi.org/10.14740/jocmr2926w mortality for both men and women in the United States (US), with one in every four deaths being attributed to heart disease [1]. Of the 370,000 deaths caused by coronary artery disease (CAD) every year, about $20.8 \%$ are Hispanics [1]. Compared with non-Hispanic whites (NHW), Hispanics display a worse CVD risk profile including higher rates of central obesity, physical inactivity, type II diabetes mellitus, atherogenic dyslipidemia, lower levels of education, and lower socioeconomic status. All these factors have been associated with increased CVD morbidity and mortality in the general population [2].

The United States Census estimates that, of the 315 million people living in the US, 54 million (17\%) are Hispanics, becoming the largest ethnic minority in the country [3]. However, Hispanics are underrepresented in studies describing racial or ethnic disparities in the care of patients with CVDs [4]. Within the US, disparities in the burden of CVD persist among racial and ethnic sub-populations and substantial heterogeneity has been observed in the trends across geographic regions. Data on CAD and acute myocardial infarction (AMI) specific to Hispanic subgroups are limited to Mexican Americans and lacking in other subgroups (e.g., Cuban Americans, Dominicans and Puerto Ricans), as reflected in the 2010 American Heart Association (AHA) statistics [4]. Nevertheless, the true burden of CVD in the growing immigrant populations from Mexico and other Spanish-speaking countries has not been adequately defined [5]. Most of the earlier studies on Hispanics/Latinos residing in US largely involve Mexican-Americans or examined Hispanics/Latinos residing in the US as a homogenous group with only a few studies has included other Hispanic/Latino background groups. Usually these studies were limited in sample size or included only a small number of Hispanic/Latino individuals. Moreover, the studies that included diverse Hispanics/Latinos suggested marked heterogeneity in risk factor prevalence within this population [6]. Therefore, there is need for a specific epidemiologic analysis for different Hispanic subpopulations [7]. This is particularly important because Hispanics comprise a heterogeneous group that is bound by common language rather than by uniform environmental origin [5].

Puerto Rican Hispanics are one of the fastest growing groups in the US, currently being the second largest Hispanic group only behind the Mexican-American Hispanics [8]. Only one previous study has described the epidemiological profile of patients admitted with an acute coronary syndrome (ACS) in Puerto Rico [9]. However, this study only included data for the year 2007 and was restricted to patients in the greater San 
Table 1. Puerto Rico Cardiovascular Surveillance Study. Baseline Patient Characteristics

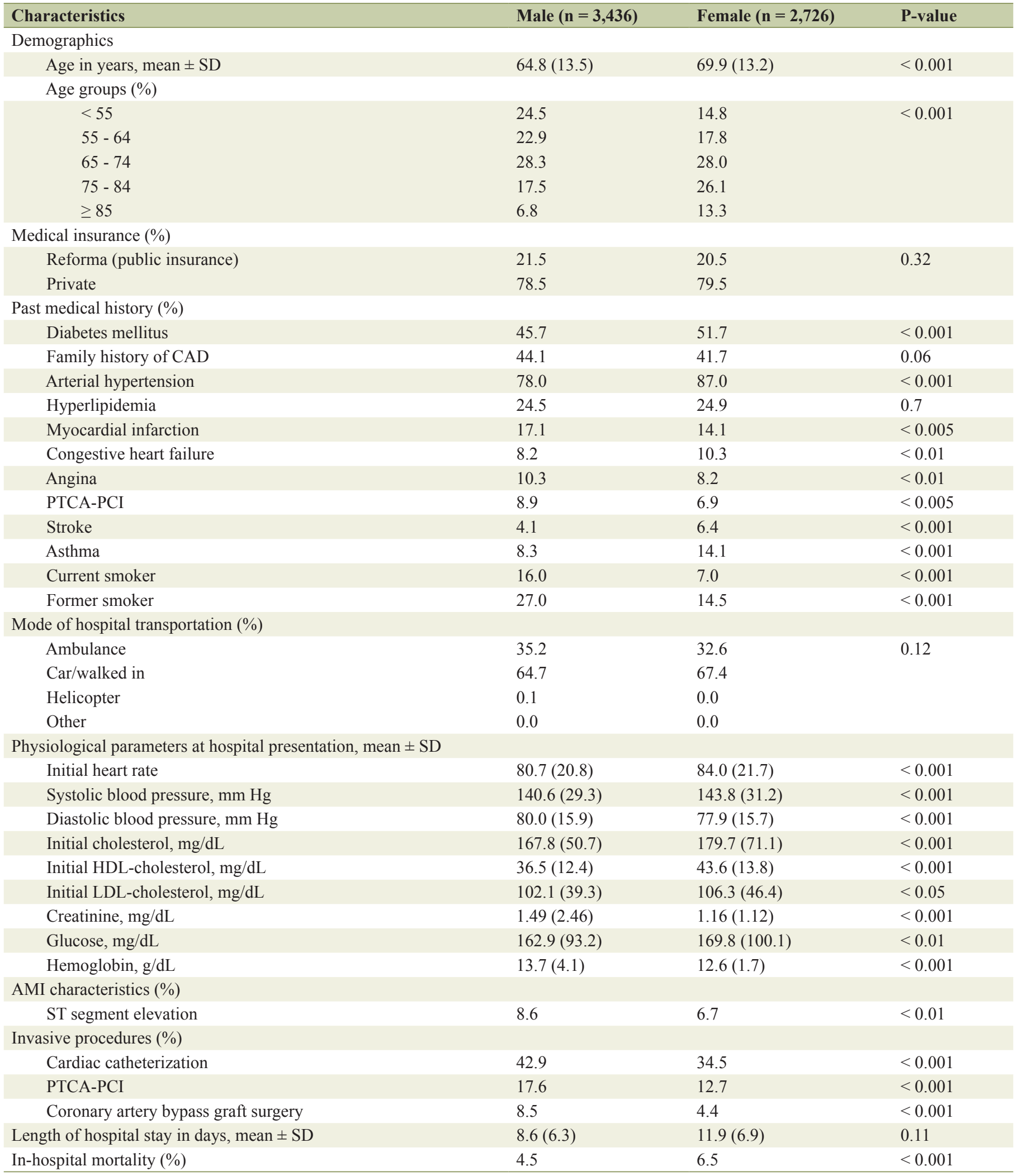

SD: standard deviation; CAD: coronary artery disease; AMI: acute myocardial infarction; PTCA: percutaneous transluminal coronary angioplasty; $\mathrm{PCl}$ : percutaneous coronary intervention. 
Table 2. Medications Administered Within $24 \mathrm{H}$ of Admission, During Hospitalization, and at Discharge

\begin{tabular}{|c|c|c|c|c|c|c|c|c|c|}
\hline \multirow{2}{*}{ Medication } & \multicolumn{3}{|c|}{ Within 24 h } & \multicolumn{3}{|c|}{ During hospitalization } & \multicolumn{3}{|c|}{ At discharge } \\
\hline & Male (\%) & Female (\%) & P-value & Male (\%) & Female (\%) & P-value & Male (\%) & Female (\%) & P-value \\
\hline ACEi/ARB & 52.9 & 49.2 & $<0.01$ & 55.1 & 48.8 & $<0.001$ & 40.6 & 37 & $<0.01$ \\
\hline Plavix & 64.9 & 64.4 & 0.71 & 72.2 & 70.1 & 0.06 & 48 & 44.7 & $<0.05$ \\
\hline Beta blockers & 58.7 & 56 & $<0.05$ & 67.3 & 63.1 & $<0.001$ & 42.4 & 38 & $<0.001$ \\
\hline
\end{tabular}

Sample: males $(3,436)$, females $(2,726)$. ACEi: angiotensin-converter enzyme inhibitor; ARBs: angiotensin receptor blockers; LMWH: low-molecularweight heparin; n/a: not applicable.

Juan area. In this paper, we expand the limited literature available to evaluate the epidemiological profile of patients with ACS across the island and over a longer time span. The aim of this study was to describe the epidemiological profile of Hispanic patients admitted with an acute coronary infarction in 19 hospitals distributed throughout Puerto Rico during the years 2007, 2009 and 2011.

\section{Methods}

\section{Data source and study design}

We conducted a secondary analysis of data collected in $19 \mathrm{Pu}-$ erto Rican Hospitals as part of the Puerto Rico Cardiovascular Surveillance Study. Information with International Classification of Diseases, Ninth Revision, Clinical Modification (ICD9-CM) from medical records was obtained by trained medical personnel. Patients with AMI admitted with an initial diagnosis of ACS (ICD 9 code: 410) or chest pain (ICD 9 code: 786.5 ) in these Hospitals during years 2007, 2009 and 2011 were included in the study. The diagnosos of an acute coronary syndrome was confirmed by the trained record reviewers. We used the information compiled in the dataset until June 30, 2014. No exclusion criteria were applied to the study population. The patient information was entered into a data instrument created using a secure web-based application (REDCap) [10]. Variables selected for analysis included age, gender, health insurance, past medical history, mode of transportation to the hospital, AMI characteristics, cardiac procedures, physiological parameters, in-hospital mortality and medications received during the hospitalization.

\section{Statistical analysis}

Descriptive analyses for the distribution of myocardial infarction indicators were performed. Continuous variables were presented using central tendency and dispersion measures (mean \pm standard deviation). Normality tests were performed for the continuous variables. Frequency distributions and percentages were used to describe categorical variables. Stratification by sex was performed to evaluate gender differences in demographics, in-hospital mortality, clinical history, risk factors, symptoms, AMI characteristics, cardiac procedures performed, acute phase treatment (medications employed), physiological parameters and secondary prevention treatment. Chi-squared test and $t$-test were used to compare proportions and means (assuming normality of data distribution) of variables of interest for gender groups. For all tests, a P-value $<$ 0.05 was considered statistically significant.

All statistical analyses were performed using STATA version 14 (available at: Url://www.stata.com). The Committee for the Protection of Human Subjects at each participating hospital approved this study.

\section{Results}

\section{Demographics and CVD risk factors}

During the study period, a total of 6,162 patients were hospitalized with AMI in 19 participating hospitals in Puerto Rico. Most patients were men $(55.8 \%)$ and had a private insurance $(79.0 \%)$

The mean age of patients diagnosed with AMI was $67.0 \pm$ 13.6 years old, women being older than men $(69.9 \pm 13.2$ vs. $64.8 \pm 13.5 ; \mathrm{P}=0.0001$ ) (Table 1). Age-group distribution suggests that men are suffering from an AMI at an earlier age with almost one-third of all females being older than 75 years old at presentation $(\mathrm{P}=0.0001)$ (Table 1$)$.

Significant differences in past medical history were found between genders (Table 1). Compared to men, women were more likely to have a history of diabetes mellitus, arterial hypertension, congestive heart failure, stroke and bronchial asthma $(\mathrm{P}<0.01)$. Nevertheless, more men have had a history of smoking cigarettes, angina, prior AMI and percutaneous transluminal coronary angioplasty (PTCA)-percutaneous coronary intervention (PCI) $(\mathrm{P}<0.01)$. No differences in hyperlipidemia and family history of CAD were seen among genders.

\section{Hospital presentation and AMI characteristics}

In general, most patients arrived to the hospital by car or walked in $(65.9 \%)$ with only one out of three patients being brought to the hospital by ambulance. Additionally, men were more 
affected with an ST-segment elevation myocardial infarction when compared to women $(8.6 \%$ vs. $6.7 \%, \mathrm{P}<0.01)$. Upon admission, a higher average serum LDL-cholesterol level (P $<0.05)$, creatinine, hemoglobin and diastolic blood pressure were reported in men $(\mathrm{P}<0.01)$, whereas women presented with higher HDL-cholesterol levels, heart rate, systolic blood pressure and glucose $(\mathrm{P}<0.01)$.

\section{In-hospital management}

Within $24 \mathrm{~h}$ of admission, $69.3 \%$ and $64.7 \%$ of patients were treated with aspirin and clopidogrel, respectively. However, at the time of discharge, just $53.7 \%$ and $44.7 \%$ were prescribed with these medications correspondingly. Furthermore, women were less likely to have received medications at admission, during their hospital stay and at discharge (Table 2). A low number of cardiac procedures were performed in our patient population. Approximately one out of two patients have benefited from cardiac catheterization. By contrast, 15.4\% had PCI and $6.7 \%$ had coronary artery bypass graft surgery (CABG). Compared to men, women were less likely to receive cardiac procedures $(\mathrm{P}<0.01)$ (Table 1$)$.

\section{LOS and in-hospital mortality}

The average LOS was 6.6 days $( \pm 10.2)$. Significant gender differences were observed when we analyzed the in-hospital mortality rate. Although the overall in-hospital mortality rate was $5.4 \%$, women were more likely to die than men $(6.5 \%$ vs. $4.5 \%, \mathrm{P}<0.01)$.

No gender differences were observed in LOS, mode of transportation and primary insurance held $(\mathrm{P}>0.05)$ (Table 1).

\section{Discussion}

This study provides the most recent information regarding the clinical profile and management of AMI in Puerto Rican residents. Gender disparities in medical management and inhospital mortality were also demonstrated.

The mean age at diagnosis of AMI was 67.0 years for $\mathrm{Pu}-$ erto Rican patients similar to that reported by Fang et al in 2010 (67 years) and lower than those reported in the Worcester Heart Attack Study (WHAS) in 2005 (71 years) [11, 12]. The mean ages of presentation for women and men were 69.9 and 64.8 years, respectively. Hence, women present with AMI approximately 5 years after men. A similar trend was reported by Nedkoff et al in 2015 (67.4 and 61.7 years), whereas in Fang et al (2010), the gap between female and male age at presentation was wider (71 and 64 years) $[11,13]$.

In terms of patient's comorbidities prior to initial AMI event, our data show that women were more likely to have a history of diabetes mellitus $(51.7 \%)$, arterial hypertension $(87.0 \%)$, congestive heart failure (CHF) $(10.3 \%)$, and stroke $(6.4 \%)$. On the other hand, male patients were more likely to be current smokers $(16.0 \%)$ and former smokers $(27.0 \%)$. In terms of hyperlipidemia and family history of heart disease, the results were not statistically significant between genders. These results are similar to those observed in the WHAS (2005) where female patients were more likely to suffer from diabetes mellitus, hypertension, stroke, and CHF; however, no significant difference was found in other comorbidities [12]. In a similar way, Zucker et al (1997) found women to be more likely to suffer from diabetes mellitus $(25 \%)$ and hypertension (55\%) when compared to men [14]. Nedkoff et al (2015) also found women to have a higher prevalence of comorbid conditions such as diabetes mellitus, hypertension, and $\mathrm{CHF}$ [13]. These findings warrant further research into the relationship between an increased risk factor burden for Puerto Rican women presenting with an AMI and worse clinical outcomes, such as in-hospital mortality.

The majority $(65.9 \%)$ of the patients included in our study used a car or walk-in as mode of transportation with no gender differences. The reason behind this preference in unknown at this time and is a theme which needs to be deeply investigated. It could be related to unavailability of ambulance at the time of event or a decision made in search of shorter time to medical care. In 2010, Song published an article comparing modes of transportation in case of an AMI in terms of efficacy and patient selection [15]. His results were very similar to ours in that $62.7 \%$ of the patients arrived in private transportation whereas only $37.3 \%$ arrived on ambulance. According to his study, if one considers emergency medical services (EMS) to be part of medical care, then it was faster than arrival by private transport to a health care facility. However, if arrival to hospital is the main goal, then using a private transport is much faster and less dependent for patients and relatives. Nevertheless, when using administration of reperfusion therapy as a more rigorous measure of treatment initiation, then use of ambulance was associated with a significant decrease in door-to-balloon time [15]. This is an area to be investigated in order to assess if our population would absolutely benefit from increased implementation of EMS in order to reduce mortality and complications in patients presenting with AMI.

When comparing the type of AMI between males and females, our results reveal that women are more likely to present with non-ST-segment elevation myocardial infarction (NSTE$\mathrm{MI})$ when compared to men $(\mathrm{P}<0.05)$. Similar results were reported by Roberts (2014) where women were significantly more likely than men to have NSTEMI (37.5\% vs. 30.7\%) [16]. Nevertheless, Zucker et al (1997) observed no difference between gender in STEMI likelihood, but report a higher number of men with ST elevations as compared to women (OR: 1.9; CI: 1.5 - 2.3). Therefore, the gender interaction showed an increase in likelihood of women with ST elevations rather than without [14].

In terms of the in-hospital management, our study shows that Puerto Rican women admitted with a myocardial infarction were less likely to receive appropriate medical therapy or a cardiac revascularization procedure. When compared to men, the women included in our study were less likely to receive ACE inhibitors/ARBs, aspirin, beta-blockers and lipid-lowering agents at any point in the hospitalization. There was no statistically significant difference between males and females in the use of clopidogrel. These findings are comparable to those 
of a study performed by Jneid et al that also found that women presenting with AMI were less likely than male counterparts to receive aspirin and beta-blockers within $24 \mathrm{~h}$ of arrival [17]. A similar pattern was noticed when analyzing the data regarding the cardiac procedures performed during hospitalization, in which women were less likely to undergo a cardiac catheterization, PTCA or CABG. These findings are consistent with those reported by Fang et al's study in which he noted that even though cardiac catheterizations, CABG, PTCA, and use of thrombolytic therapy have increased through time, a higher percentage of men received these procedures when compared to women [11]. However, despite these gender differences, it is still worthwhile to note that compared to the only previously published study in Puerto Rico [18], there is a persistent underutilization of guideline-directed pharmacologic therapy among Puerto Rican patients with an AMI.

Our results reveal that the mean LOS for patients diagnosed and admitted with an AMI was 6.3 days in females and 6.9 days for men without a statistically significant difference between genders. Our results contrast with those reported by Fang et al and WHAS where the LOS was approximately 4 and 5.3 days, respectively, both lower than those seen in our data $[11,12]$. Saczynski et al (2010) also show decreased LOS (5 days) compared to our data and revealed a $30 \%$ decline from previous data obtained in 1995 (7.2 days) in the Worcester area (MA) [19]. These studies show no difference in LOS between genders but do demonstrate an association with age where the median LOS increased with older females as well as development of complications such as stroke or cardiogenic shock [11, 20]. Although gender was not an important factor determining the LOS in our population, we must emphasize that Puerto Ricans admitted with AMI have an LOS above the national average (4.8 days). This fact should bring further attention and merits future studies to determine which factors are affecting the LOS in this particular population.

Since 1984, the number of CVD-related deaths has been greater in women than men. In $2009,51 \%$ of the deaths attributed to CVD in US were women; the death rate of CVD was 236 with 190.4 for white women and 267.9 for black women [6]. Puerto Ricans admitted with AMI show the same profile when compared in-hospital mortality rate, since women were more likely to die than men $(6.5 \%$ vs. $4.55 \%, \mathrm{P}<0.001)$. This trend has been observed since 1768, when Heberden described for the first time a gender-specific difference in CVD [21]. In 1999 , Vaccarino et al abstracted data on over 155,000 women and 229,000 men who had been entered into the National Registry of Myocardial Infarction [22]. In this study, more women died than men under similar circumstances (16.7\% vs. $11.5 \%)$. Moreover, when examining age groups separately, he found that under 50 years of age mortality was $6.1 \%$ in women and $2.9 \%$ in men. Quite the reverse, he found no significant difference in mortality between men and women after age 74 .

This study has some limitations. First, AMI patients admitted with a different initial diagnosis other than ACS or chest pain were not included. Second, our data represent Hispanics and consequently, our results may not be generalized to other ethnic/racial groups. However, there are important strengths to the study, including the large sample size and represented data of real-life management practices on Hispanics patients hospitalized with AMI in Puerto Rico.

\section{Conclusion}

Our study shows significant gender disparities in Hispanic population as well as some deficiencies in clinical management and outcomes of AMI. To our knowledge, this is the broader study conducted so far in the island that describes the epidemiological profile in patients hospitalized with AMI. As part of Puerto Rico Cardiovascular Surveillance Study, it represents an expandable source of information with a strong update potential over the several next years. Knowledge about the epidemiological behavior of this population is critical, so hospital can establish and implement preventive measures, reduce gender disparities, and raise awareness of coronary heart disease among Puerto Rican populations, thus, impacting clinical management and promoting public health initiatives to improve the care provided to Puerto Rican patients with AMI.

\section{Acknowledgments}

Research reported in this publication was supported by the National Institute on Minority Health and Health Disparities of the National Institutes of Health under Award Numbers 5S21MD000242, 5S21MD00138, 2U54MD007587 and G12MD007600 and the University of Puerto Rico, School of Medicine. The content is solely the responsibility of the authors and does not necessarily represent the official views of the National Institutes of Health. A special acknowledgement was given to Angel Pena and Marisela Irizarry for the collaboration during the development of this research.

\section{Conflicts of Interest}

This article content has no conflicts of interest.

\section{Author Note}

All authors take responsibility for all aspects of the reliability and freedom from bias of the data presented and their discussed interpretation.

\section{References}

1. CDC, NCHS. Underlying Cause of Death 1999-2013 on CDC WONDER Online Database, released 2015. Data are from the Multiple Cause of Death Files, 1999-2013, as compiled from data provided by the 57 vital statistics jurisdictions through the Vital Statistics Cooperative Program.

2. United States Census Bureau. Facts for Features: Hispanic Heritage Month 2014: Sept 15- Oct 15. Accessed 
April 10, 2015. http://www.census.gov/newsroom/factsfor-features/2014/cb14-ff22.html.

3. Medina-Inojosa J, Jean N, Cortes-Bergoderi M, LopezJimenez F. The Hispanic paradox in cardiovascular disease and total mortality. Prog Cardiovasc Dis. 2014;57(3):286292.

4. Krim SR, Vivo RP, Krim NR, Cox M, Hernandez AF, Peterson ED, Fonarow GC, et al. Regional differences in clinical profile, quality of care, and outcomes among Hispanic patients hospitalized with acute myocardial infarction in the Get with Guidelines-Coronary Artery Disease (GWTG-CAD) registry. Am Heart J. 2011;162(6):988995 e984.

5. Cortes-Bergoderi M, Goel K, Murad MH, Allison T, Somers VK, Erwin PJ, Sochor O, et al. Cardiovascular mortality in Hispanics compared to non-Hispanic whites: a systematic review and meta-analysis of the Hispanic paradox. Eur J Intern Med. 2013;24(8):791-799.

6. Go AS, Mozaffarian D, Roger VL, Benjamin EJ, Berry JD, Blaha MJ, Dai S, et al. Heart disease and stroke statistics - 2014 update: a report from the American Heart Association. Circulation. 2014;129(3):e28-e292.

7. Allison MA, Budoff MJ, Wong ND, Blumenthal RS, Schreiner PJ, Criqui MH. Prevalence of and risk factors for subclinical cardiovascular disease in selected US Hispanic ethnic groups: the Multi-Ethnic Study of Atherosclerosis. Am J Epidemiol. 2008;167(8):962-969.

8. United States Census Bureau. The Hispanic Population in the United States: 2012. http://www.census.gov/population/hispanic/data/2012.html.

9. Zevallos JC, Yarzebski J, Banchs H, Gonzalez-Sanchez JA, Mattei H, Goldberg RJ, Gonzalez Mdel C, et al. Gender disparities in Puerto Ricans hospitalized with an initial acute myocardial infarction: A population-based perspective. P R Health Sci J. 2012;31(4):192-198.

10. Harris PA, Taylor R, Thielke R, Payne J, Gonzalez N, Conde JG. Research electronic data capture (REDCap) - a metadata-driven methodology and workflow process for providing translational research informatics support. J Biomed Inform. 2009;42(2):377-381.

11. Fang J, Alderman MH, Keenan NL, Ayala C. Acute myocardial infarction hospitalization in the United States, 1979 to 2005. Am J Med. 2010;123(3):259-266.

12. Floyd KC, Yarzebski J, Spencer FA, Lessard D, Dalen JE,
Alpert JS, Gore JM, et al. A 30-year perspective (19752005) into the changing landscape of patients hospitalized with initial acute myocardial infarction: Worcester Heart Attack Study. Circ Cardiovasc Qual Outcomes. 2009;2(2):88-95.

13. Nedkoff L, Atkins E, Knuiman M, Sanfilippo FM, Rankin J, Hung J. Age-specific gender differences in long-term recurrence and mortality following incident myocardial infarction: a population-based study. Heart Lung Circ. 2015;24(5):442-449.

14. Zucker DR, Griffith JL, Beshansky JR, Selker HP. Presentations of acute myocardial infarction in men and women. J Gen Intern Med. 1997;12(2):79-87.

15. Song L, Yan H, Hu D. Patients with acute myocardial infarction using ambulance or private transport to reach definitive care: which mode is quicker? Intern Med J. 2010;40(2):112-116.

16. Roberts BH. Gender-specific aspects of cardiovascular disease. R I Med J (2013). 2014;97(2):19-22.

17. Jneid H, Fonarow GC, Cannon CP, Hernandez AF, Palacios IF, Maree AO, Wells Q, et al. Sex differences in medical care and early death after acute myocardial infarction. Circulation. 2008;118(25):2803-2810.

18. Zevallos JC, Yarzebski J, Gonzalez JA, Banchs HL, Garcia-Palmieri M, Mattei H, Ayala J, et al. Incidence, inhospital case-fatality rates, and management practices in Puerto Ricans hospitalized with acute myocardial infarction. P R Health Sci J. 2013;32(3):138-145.

19. Saczynski JS, Lessard D, Spencer FA, Gurwitz JH, Gore JM, Yarzebski J, Goldberg RJ. Declining length of stay for patients hospitalized with AMI: impact on mortality and readmissions. Am J Med. 2010;123(11):1007-1015.

20. Spencer FA, Lessard D, Gore JM, Yarzebski J, Goldberg RJ. Declining length of hospital stay for acute myocardial infarction and postdischarge outcomes: a communitywide perspective. Arch Intern Med. 2004;164(7):733740.

21. Heberden W. Some account of a disorder of the breast. Med Trans Coll Phys. 1772;2:59-67.

22. Vaccarino V, Parsons L, Every NR, Barron HV, Krumholz HM. Sex-based differences in early mortality after myocardial infarction. National Registry of Myocardial Infarction 2 Participants. N Engl J Med. 1999;341(4):217225 . 